\title{
Amonificación de la Panca de Maíz (Zea mays L) con Tres Niveles de Urea para la Mejora de su Digestibilidad
}

\author{
Amonification of Corn Stover ( $Z_{\text {Ea }}$ mays L) With Three Levels of Urea to \\ IMPROVE ITS DigESTIBILITY
}

Shirley Castellanos ${ }^{1}$, Jorge Gamarra ${ }^{1}$, Carlos Gómez ${ }^{1,2}$, Melisa Fernández ${ }^{1}$

\section{Resumen}

El objetivo del presente estudio fue evaluar el efecto de la urea $(46 \%$ de N) sobre la calidad nutricional de la panca de maíz proveniente de las zonas de Chilampa, La Villa, Miramar y La Querencia, de la Asociación de Ganaderos de la Irrigación San Felipe, situada en la provincia de Huaura, Lima (Perú). Se trabajó con tres niveles de urea $(0,3$ y $6 \%$ ) en forma de solución acuosa sobre la panca de maíz almacenada herméticamente en bolsas plásticas durante un periodo de 14 días. Se evaluaron las siguientes variables: proteína cruda (PC\%), fibra detergente neutro (FDN\%), digestibilidad in vitro de la materia seca (DIVMS\%), digestibilidad in vitro de la fibra detergente neutro (DIVFDN\%), ingesta potencial de materia seca (\%PV en MS) y valor relativo del forraje (VRF), con un diseño de bloques completamente al azar con tres repeticiones. Los resultados para los tratamientos 0,3 y $6 \%$ de urea fueron: $84.66,70.01$ y $67.78 \%$ de MS; 5.08, 8.02 y $12.92 \%$ de PC; 77.31, 76.89 y 74.84\% de FDN; 59.90, 61.17 y 66.59\% de DIVMS; 48.15, 50.97 y $55.41 \%$ de DIVFDN; $1.55,1.56$ y $1.60 \%$ de ingesta potencial de materia seca; y 72.09 , 74.03 y 82.81 de VRF, respectivamente. No se encontraron diferencias significativas entre las procedencias de la panca de maíz, ni para la DIVFDN entre los niveles de urea aplicada; sin embargo, hubo diferencia significativa $(\mathrm{p}<0.05)$ para el contenido de $\mathrm{PC}$, FDN, DIVMS, ingesta potencial de materia y VRF entre los niveles de urea.

Palabras clave: amonificación, panca de maíz, urea, digestibilidad

\section{Abstract}

The aim of this study was to evaluate the effect of urea $(46 \% \mathrm{~N})$ on the nutritional quality of corn stover from the areas of Chilampa, La Villa, Miramar and La Querencia of the Cattle Association Irrigación San Felipe, located in Huaura, Lima (Peru). Three levels of urea $(0,3$ and $6 \%)$ in an aqueous solution over a period of 14 days were applied

\footnotetext{
${ }^{1}$ Departamento de Nutrición, Facultad de Zootecnia, Universidad Nacional Agraria La Molina, Lima, Perú

2E-mail: cagomez@lamolina.edu.pe
}

Recibido: 26 de julio de 2016

Aceptado para publicación: 16 de noviembre de 2016 
and the forage was stored in sealed plastic bags. The variables evaluated were crude protein content $(\mathrm{CP} \%)$, neutral detergent fiber content (NDF\%), in vitro digestibility of dry matter (IVDDM\%), in vitro digestibility of neutral detergent fiber (IVNDFD\%), intake potential of dry matter (\%PV DM) and relative feed value (RFV). Randomized complete block design with three replications was used. The results for 0,3 and $6 \%$ urea were: 84.66, 70.01 and $67.78 \% \mathrm{DM} ; 5.08,8.02$ and $12.92 \% \mathrm{CP} ; 77.31,76.89$ and $74.84 \% \mathrm{NDF}$; $59.90,61.17$ and $66.59 \%$ IVDMD; $48.15,50.97$ and $55.41 \%$ IVNDFD; $1.55,1.56$ and $1.60 \%$ potential intake of dry matter; and $72.09,74.03$ and $82.81 \mathrm{FRV}$ respectively. The results of the analysis showed no difference between the source of corn stover or for IVNDFD between the urea levels; however, there was significant difference $(p<0.05)$ of CP content, NDF, IVDMD, potential intake of dry matter and the FRV between the levels of urea.

Key words: ammonification, corn stover, urea, digestibility

\section{INTRODUCCIÓN}

En la costa peruana, durante los meses de invierno escasean los forrajes de buena calidad como el maíz, lo cual ocasiona una elevación de los precios. Por este motivo, los ganaderos recurren a residuos de cosecha como la panca de maíz para cubrir la menor disponibilidad de forrajes. La mayoría de residuos de cosechas agrícolas, como el rastrojo de maíz, presentan un bajo contenido de proteína $(<5 \%)$, un alto contenido de carbohidratos estructurales y un alto componente de lignina ( $70 \%$ de pared celular) (Escobar y Parra, 1980), que hace que estos insumos provean una baja proporción de energía digestible a los herbívoros (Flores, 1990). Sin embargo, se puede mejorar este aporte de energía a través de procedimientos físicos y químicos, considerando la peculiar fisiología digestiva fermentativa del rumiante (Owen, 1976; Jackson, 1978).

La amonificación con urea es una técnica química que le permite al ganadero hacer un mejor uso de los residuos de cosecha al incrementar su valor nutricional. El tratamiento con urea ha demostrado que mejora el valor nutritivo de los forrajes de baja calidad por el efecto del ion amonio en los carbohidratos de la pared celular (Chesson et al., 1983), siendo el efecto final un aumento de la digestibilidad in vitro de la materia seca (DIVMS), de la digestibilidad in vitro de la fibra detergente neutro (DIVFDN), del contenido de nitrógeno y del consumo de materia seca (MS) (Oji et al., 1977) y la productividad de los animales (Hamad et al., 2010). Además, se incrementa los niveles de proteína debido a la adición de nitrógeno proveniente de la urea (Sánchez et al., 2015). Tesfaye et al. (2006) trataron la panca de maíz (91.6\% de MS y $4.0 \%$ de PC) con varios niveles de urea encontrando diferencias favorables en el contenido de proteína cruda (PC). En este sentido, el objetivo de la presente investigación fue evaluar un protocolo de tratamiento físico/químico (amonificación con urea) de la panca de maíz de la Asociación de Ganaderos de la Irrigación San Felipe (AGISF), para mejorar su calidad nutricional.

\section{Materiales y Métodos}

\section{Ubicación del Estudio}

El estudio se realizó en la Asociación de Ganaderos de la Irrigación San Felipe (AGISF), situada en el distrito La Vegueta, provincia de Huaura, Lima, Perú, entre julio y setiembre de 2014. Los tratamientos con urea de las muestras de panca de maíz se realizaron en el Laboratorio de Evaluación Nutricional de Alimentos (LENA) de la Uni- 
versidad Nacional Agraria La Molina (UNALM), Lima.

\section{Encuesta}

El diseño del estudio tuvo como base los resultados de una encuesta realizada a 30 ganaderos de los 151 socios de la AGISF, de las cuatro zonas que la componen, para conocer, entre otros aspectos, la forma de uso de la panca de maíz (molida, picada, entera), el racionamiento promedio a los animales y calidades, así como el nivel de conocimiento respecto al tratamiento de forrajes.

\section{Muestras de Panca de Maíz}

Se recolectaron muestras de panca de maíz que estaban siendo utilizadas por los ganaderos encuestados. Las muestras del grupo I estuvieron conformadas por panca molida de la zona de Chilampa y de La Querencia, las del grupo II (debido a su cercanía) por muestras de panca molida de la zona de La Villa y Miramar, y las del grupo III por panca entera de la zona de Chilampa.

De cada grupo se tomaron nueve muestras de $2 \mathrm{~kg}$ para los tratamientos respectivos. Se determinó el tamaño de partícula de la panca de maíz de las tres procedencias utilizando el separador de partícula Penn State (Lammers et al., 1996).

\section{Tratamientos Experimentales}

Las nueve muestras de panca de maíz de cada grupo se dividieron en tres subgrupos, recibiendo tratamientos con tres niveles de urea $(0,3$ y $6 \%)$ en tres repeticiones. $\mathrm{La}$ panca entera del grupo III fue molida para efectos del estudio. Para el tratamiento con 3 y $6 \%$ de urea se utilizaron 60 y $120 \mathrm{~g}$ de urea para $2 \mathrm{~kg}$ de panca molida (tal como ofrecida), respectivamente.

Antes de realizar los tratamientos con urea, se determinó el nivel de humedad de las muestras en una estufa de aire circulante a $105^{\circ} \mathrm{C}$ por $8 \mathrm{~h}$ (AOAC, 2005), para calcu- lar la cantidad de agua necesaria en la dilución de la urea y poder alcanzar una humedad del 30\% según recomendaciones de DíasDa-Silva et al. (1988). Es así que para $2 \mathrm{~kg}$ de panca con $14.8 \%$ de humedad inicial, fue necesario añadir $434 \mathrm{ml}$ de agua para diluir la urea y llegar a obtener una humedad teórica de $30 \%$.

Las muestras de panca de maíz recibieron sus respectivos tratamientos con ayuda de un aspersor y fueron almacenadas herméticamente en bolsas de plástico durante 14 días. La duración del tratamiento se basó de acuerdo a la temperatura ambiental de $22.5^{\circ} \mathrm{C}$ en promedio, siguiendo las recomendaciones de Sundstol et al. (1978). Luego de este periodo, el contenido de las bolsas de plástico fue transferido a bolsas de papel para ser secadas en una estufa de aire circulante a $60^{\circ} \mathrm{C}$ durante cuatro días, determinándose el porcentaje de materia seca. La molienda de las muestras se hizo en un molino Willey de tijeras con una malla de $1 \mathrm{~mm}$.

\section{Variables Evaluadas}

El contenido de proteína cruda (PC) se evaluó por el método semi-micro Kjeldahl (AOAC, 2005) y el contenido de fibra detergente neutro (FDN) se basó en lo estipulado por ANKOM (2005). A su vez, tanto la digestibilidad in vitro de la materia seca (DIVMS) como la digestibilidad in vitro del FDN (DIVFDN) se evaluó siguiendo el procedimiento de Tilley y Terry, modificado por Van Soest (Goering y Van Soest, 1972).

La ingesta potencial de materia seca (\%PV en MS) y el valor relativo del forraje (VRF) se calcularon usando la siguiente fórmula (Jeranyama y García, 2004): VRF = (\%DIVMS) x (Ingesta [\%PV en MS]/1.29, donde $\mathrm{PV}$ es peso vivo e Ingesta (\%PV en $\mathrm{MS})=120 / \% \mathrm{FDN}$.

El VRF es un índice que supone una valoración objetiva de la calidad del forraje que refleja el potencial de ingestión de materia seca y su digestibilidad, permitiendo la cla- 
Cuadro 1. Calidad del forraje según su valor relativo del forraje

\begin{tabular}{cl}
\hline $\begin{array}{c}\text { Valor relativo del } \\
\text { forraje(VRF) }\end{array}$ & Calidad \\
\hline$>155$ & Excelente \\
$125-155$ & Primera \\
$103-124$ & Segunda \\
$87-102$ & Tercera \\
$75-86$ & Cuarta \\
$<75$ & Quinta \\
\hline
\end{tabular}

sificación en forrajes en calidades, tal como se muestra en el Cuadro 1 (FEDNA, 2014).

\section{Diseño Estadístico}

Se empleó un diseño de bloques completamente al azar con error de muestreo, teniendo tres niveles de urea como tratamiento y tres procedencias de panca de maíz como bloques con tres repeticiones por tratamiento. Se utilizó la prueba de Duncan, con un nivel de significancia de 0.05 para la comparación entre los promedios de los tratamientos. Para el análisis de varianza, se utilizó el programa estadístico SAS v. 9.1.

\section{Resultados y Discusión}

\section{Encuesta}

E1 90\% de los ganaderos usaba la panca como un componente de la dieta forrajera para las vacas y animales de recría, pudiendo ser entera $(47 \%)$ o molida ( $43 \%$ ). La razón de estas diferencias podría deberse al menor costo de la panca entera (S/. 97 por tonelada métrica) respecto a la molida $(\mathrm{S} /$. 256). Solo el $17 \%$ de los ganaderos que compran panca entera logra procesarla, sea moliéndola o picándola.
La limitación de uso de la panca de maíz se debe a la baja disponibilidad de equipos de molienda y a los altos costos de servicio de molienda. Por otro lado, los ganaderos no usan técnicas convencionales para conservar los forrajes ni tratamientos de residuos fibrosos para mejorar su calidad.

\section{Efecto sobre la Composición Química}

\section{Contenido de proteína cruda (PC)}

Los valores promedios del contenido de MS y PC en base seca para la panca de maíz de las tres procedencias y que recibieron tratamientos con los tres niveles de urea se muestran en el Cuadro 2. Se encontró diferencia significativa $(p<0.01)$ en el contenido de PC entre los niveles de urea aplicado a la panca de maíz; no obstante, no hubo diferencia debido a la procedencia de la panca, lo que indica que se podría emplear panca de maíz de cualquier procedencia para mejorar su contenido de PC con la técnica de la amonificación con urea.

La panca de maíz tratada con $6 \%$ de urea presentó mayor $(\mathrm{p}<0.05)$ contenido de PC (12.92\%) que los demás tratamientos. Los valores obtenidos de PC fueron similares a los reportados por Arriarán (1989), quien reportó contenidos de 9.81 y $12.30 \%$ de PC con 3 y $5 \%$ de urea, respectivamente. Por otro lado, Tesfaye et al. (2006) reportaron mayores valores de PC $(14.90,15.92$ y $16.87 \%$ ) al someter la panca de maíz a 4,5 y $6 \%$ de urea durante 1,2 y 3 semanas a 25 ${ }^{\circ} \mathrm{C}$. Esto pudo deberse al mayor tiempo de amonificación y a las diferencias en la calidad inicial de la panca de maíz.

El $84.66 \%$ de MS para el grupo sin urea fue menor al $92.44 \%$ reportado por Arriarán (1989), posiblemente debido a la calidad inicial de la panca, variedad del forraje, época de cosecha, etc. Por otro lado, Elizondo (1998) encontró que el nivel de humedad no tuvo un efecto significativo sobre la MS, PC, FDN, FDA y nitrógeno no proteico (NNP) al 
Cuadro 2. Efecto del tratamiento de la panca de maíz con tres niveles de urea (\%) sobre el contenido de proteína cruda (PC) y fibra detergente neutro (FDN) en base seca (MS)

\begin{tabular}{lcccccccccc}
\hline & \multicolumn{3}{c}{$0 \%$} & \multicolumn{3}{c}{$3 \%$} \\
\cline { 2 - 10 } Procedencia & $\begin{array}{l}\text { MS } \\
(\%)\end{array}$ & $\begin{array}{c}\text { PC } \\
(\%)\end{array}$ & $\begin{array}{c}\text { FDN } \\
(\%)\end{array}$ & $\begin{array}{c}\text { MS } \\
(\%)\end{array}$ & $\begin{array}{c}\text { PC } \\
(\%)\end{array}$ & $\begin{array}{c}\text { FDN } \\
(\%)\end{array}$ & $\begin{array}{c}\text { MS } \\
(\%)\end{array}$ & $\begin{array}{c}\text { PC } \\
(\%)\end{array}$ & $\begin{array}{c}\text { FDN } \\
(\%)\end{array}$ \\
\hline Chilampa 1 & 85.80 & 5.07 & 76.73 & 69.19 & 7.87 & 76.33 & 67.82 & 12.63 & 74.67 \\
Villa-Miramar & 85.18 & 5.03 & 77.73 & 71.57 & 7.97 & 76.83 & 67.55 & 12.97 & 74.87 \\
Chilampa 2 & 82.99 & 5.13 & 77.47 & 69.26 & 8.23 & 77.50 & 67.97 & 13.17 & 75.00 \\
\hline Promedio & 84.66 & $5.08^{\mathrm{c}}$ & $77.31^{\mathrm{a}}$ & 70.01 & $8.02^{\mathrm{b}}$ & $76.89^{\mathrm{a}}$ & 67.78 & $12.92^{\mathrm{a}}$ & $74.84^{\mathrm{b}}$ \\
\hline a,b,c Promedios con & letras diferentes dentro de PC $\mathrm{FDN}$ presentan diferencias significativas (p<0.05)
\end{tabular}

Cuadro 3. Efecto del tratamiento con tres niveles de urea (\%) en la panca de maíz sobre la digestibilidad in vitro de la materia seca (DIVMS) y la digestibilidad in vitro de la fibra detergente neutro (DIVFDN) en base seca

\begin{tabular}{lcccccc}
\hline \multirow{2}{*}{ Procedencia } & \multicolumn{3}{c}{$\begin{array}{c}\text { DIVMS } \\
(\%)\end{array}$} & & \multicolumn{3}{c}{$\begin{array}{c}\text { DIVFDN } \\
(\%)\end{array}$} \\
\cline { 2 - 7 } & $0 \%$ & $3 \%$ & $6 \%$ & $0 \%$ & $3 \%$ & $6 \%$ \\
\hline Chilampa 1 & 59.87 & 62.8 & 66.37 & 47.70 & 55.88 & 54.99 \\
Villa-Miramar & 61.43 & 61.63 & 67.43 & 50.39 & 50.03 & 56.58 \\
Chilampa 2 & 58.4 & 59.07 & 65.97 & 46.36 & 47.00 & 54.68 \\
\hline Promedio & $59.90^{\mathrm{b}}$ & $61.17^{\mathrm{b}}$ & $66.59^{\mathrm{a}}$ & $48.15^{\mathrm{b}}$ & $50.97^{\mathrm{ab}}$ & $55.41^{\mathrm{a}}$ \\
\hline
\end{tabular}

a,b Promedios dentro de variables (DIVMS, DIVFDN) con letras diferentes presentan diferencias significativas $(p<0.05)$

Cuadro 4. Efecto del tratamiento con tres niveles de urea (\%) de la panca de maíz sobre la ingesta potencial de materia seca (Ingesta - \%PV en MS) y el valor relativo del forraje (VRF)

\begin{tabular}{|c|c|c|c|c|c|c|}
\hline \multirow{2}{*}{ Procedencia } & \multicolumn{2}{|c|}{$0 \%$} & \multicolumn{2}{|c|}{$3 \%$} & \multicolumn{2}{|c|}{$6 \%$} \\
\hline & Ingesta & VRF & Ingesta & VRF & Ingesta & VRF \\
\hline Chilampa 1 & 1.56 & 72.63 & 1.57 & 76.58 & 1.61 & 82.68 \\
\hline Villa-Miramar & 1.54 & 73.51 & 1.56 & 74.62 & 1.60 & 83.89 \\
\hline Chilampa 2 & 1.55 & 70.13 & 1.55 & 70.88 & 1.60 & 81.85 \\
\hline Promedio & $1.55^{\mathrm{b}}$ & $72.09^{b}$ & $1.56^{\mathrm{b}}$ & $74.03^{b}$ & $1.60^{\mathrm{a}}$ & $82.81^{\mathrm{a}}$ \\
\hline
\end{tabular}


tratar la panca de maíz molida con $5 \%$ de urea con niveles de humedad de 20 a $35 \%$ en diversos tiempos de tratamiento. En este estudio, los incrementos en el contenido de PC bajo los porcentajes de materia seca obtenidos coincidieron con Días-Da-Silva et al. (1988), quienes indican que el $30 \%$ de humedad es adecuado para tratar la panca de maíz, ya que no solo se incrementa el contenido de PC, sino que se incrementa la DIVMS y DIVFDN y se disminuye la FDN.

\section{Contenido de fibra detergente neutro $(F D N)$}

No se encontró diferencia significativa para el contenido de FDN debido a la procedencia de la panca de maíz (Cuadro 2). No obstante, la panca tratada con 0 y $3 \%$ de urea presentó un contenido de FDN similar pero superior $(\mathrm{p}<0.05)$ a la panca tratada con $6 \%$ de urea. Por otro lado, Ramírez et al. (2007) reportaron menores valores de FDN (66.0, $69.3 \%$ ) con 21 días de fermentación respecto a la panca sin tratar $(75.4 \%)$, posiblemente debido al mayor tiempo de acción fermentativa del amoniaco sobre la pared celular.

Los resultados muestran que conforme se incrementan los niveles de urea disminuyen los contenidos de FDN, coincidente con el estudio de Fuentes et al. (2001). Los resultados sugieren que la disminución de la FDN se debe a que el tratamiento con $\mathrm{NH}_{3}$ solubiliza parte de la hemicelulosa, haciendo más disponible el contenido celular. Por lo tanto, esto favorecería un mayor acceso de los microorganismos del rumen a los polisacáridos de las paredes celulares lignificadas (Chenost y Dulphy, 1987).

\section{Digestibilidad de la Panca de Maíz}

Digestibilidad in vitro de la materia seca (DIVMS)

Los valores promedios de la DIVMS para la panca de maíz se muestran en el Cuadro 3 , encontrándose diferencia altamente significativa $(p<0.01)$ entre los niveles de urea. Se observó un incremento de $11.17 \%$ de la DIVMS al aplicar 6\% de urea (66.59\%). Por otro lado, no se encontraron diferencias por efecto de la procedencia de la panca. Resultados similares fueron obtenidos por Fuentes et al. (2001), quienes reportaron valores de $66.05,71.50$ y $71.94 \%$ de DIVMS al tratar la panca de maíz molida, picada y entera, respectivamente, con $4 \%$ de amoniaco durante cuatro semanas respecto a la panca sin tratar $(64.67 \%$ de DIVMS). Similarmente, Tesfaye et al. (2006) observaron que el tratamiento con 5\% de urea incrementó la DIVMS en $7.7 \%$, respecto a la panca sin tratar.

Digestibilidad in vitro de la fibra detergente neutro (DIVFDN)

Los valores promedios de la DIVFDN para la panca de maíz se muestran en el Cuadro 3, observándose que no hubo diferencias significativas debido a la procedencia de la panca. El tratamiento control y el tratamiento con 3\% de urea obtuvieron resultados similares; sin embargo, el tratamiento con $6 \%$ de urea fue significativamente superior al tratamiento control $(\mathrm{p}<0.05)$.

\section{Ingesta Potencial de MS y Valor Relati- vo de Forraje}

Los valores promedios de la estimación de la ingesta de MS y del VRF de la panca de maíz se muestran en el Cuadro 4. La calidad forrajera de la panca de maíz amonificada fue considerada como de quinta para los tratamientos 0 y $3 \%$ y como de cuarta para el tratamiento con $6 \%$ de urea. Los resultados indican diferencia significativa $(\mathrm{p}<0.01)$ para el nivel de ingesta potencial y el VRF entre los niveles de urea aplicado a la panca de maíz; en cambio, no hubo diferencias significativas entre la procedencia del forraje.

La ingesta potencial de materia seca de la panca de maíz tratada con $6 \%$ de urea fue superior $(p<0.05)$ a los otros tratamientos. Los resultados indican que la acción solubilizante 
del ion amonio sobre los carbohidratos de la pared celular (Chesson et al., 1983) en el proceso de amonificación mejoró tanto la digestibilidad de la MS como de la FDN y, por ende, el VRF de la panca de maíz. Esto significa una mejora de su calidad forrajera del nivel más bajo de 72.09 VRF (de quinta calidad: $<75 \mathrm{VRF}$ ) al nivel superior inmediato de 82.82 VRF (de cuarta calidad: 75-86 VRF) (FEDNA, 2014).

\section{Conclusiones}

- La amonificación con 3 y $6 \%$ de urea de la panca de maíz incrementó significativamente el contenido de proteína cruda de 5.08 a 8.02 y $12.92 \%$, respectivamente; mientras que el efecto solubilizante del amonio sobre la pared celular redujo significativamente el contenido de fibra detergente neutro de la panca de maíz de 77.31 a 76.89 y $74.84 \%$, respectivamente.

- Las digestibilidades in vitro de la materia seca y de la fibra detergente neutro se incrementaron por efecto de la amonificación cuando se aplicó $6 \%$ de urea.

- El tratamiento con 6\% de urea mejoró la ingesta potencial de materia seca $(\% \mathrm{PV}$ en MS) de la panca de maíz debido a la mejora de la digestibilidad de la materia seca y de la fibra detergente neutro, mejorando el valor relativo del forraje (VRF).

\section{Literatura Citada}

1. [AOAC] International Association of Official Analytical Chemists. 2005. Official Methods of Analysis of AOAC International. $18^{\text {th }}$ ed. Arlington, Washington DC: AOAC.

2. ANKOM Technology. 2005. Neutral detergent fiber in feeds - Filter bag technique (for A2000 and A2000I). ANKOM Technology. [Internet].
Available in: https://www.ankom.com/ sites/default/files/document-files/ Method_13_NDF_A2000.pdf

3. Arriaran JC. 1989. Efecto de la panca de maíz (Zea mays L.) tratada con dos niveles de urea en la alimentación de vacas Holstein sobre la producción y tenor graso de la leche. Tesis de Ingeniero Zootecnista. Lima, Perú: Univ Nacional Agraria La Molina. $150 \mathrm{p}$.

4. Chenost M, Dulphy JP. 1987. Amelioration de la valeur alimentaire (composition, digestibilité, ingestibilité) des mauvais foins et des pailles sur les différents types de traitement. In: Demarquilly C (ed). Les Fourrages secs: recolte, traitement, utilization. París : INRA. p 199-230.

5. Chesson A, Gordon AH, Lomax JA. 1983. Substituent groups linked by alkalilabile bonds to arabinose and xylose residues of legume grass and cereal straw cell walls and their fate during digestion by rumen microorganisms. J Sci Food Agric 34 :1330-1340. doi: 10.1002/ jsfa.2740341204

6. Dias-Da-Silva AA, MascarenhaFerreira A, Guedes CVM. 1988. Effects of moisture level, treatment time and soya bean addition on the nutritive value of urea- treated maize stover. Anim Feed Sci Technol 19: 67-77. doi: 10.1016/ 0377-8401(88)90055-7

7. Elizondo I. 1998. Evaluación de tratamientos alcalinos sobre la calidad nutricional de subproductos lignocelulosicos. Tesis de Doctorado. México: Universidad de Colima.112 p.

8. Escobar A, Parra R. 1980. Procesamiento y tratamiento físico-químico de los residuos de cosecha con miras al mejoramiento de su valor nutritivo. En: Estrategias para el uso de residuos de cosecha en la alimentación animal. Turrialba, Costa Rica: Centro Agronómico Tropical de Investigación y Enseñanza. p 93-130.

9. [FEDNA] Fundación Española para el Desarrollo de la Nutrición Animal. 2014. Forrajes. [Internet]. Disponible en: http://fundacionfedna.org/forrajes 
10. Flores JA. 1990. Bromatología animal. $3^{\mathrm{a}}$ ed. México: Limusa. $930 \mathrm{p}$.

11. Fuentes J, Magaña C, Suárez L, Peña R, Rodríguez S, Ortíz de La Rosa B. 2001. Análisis químico y digestibilidad «in vitro» de rastrojo de maíz (Zea mays L) Agron Mesoamericana 12: 189-192.

12. Hamad MR, Safaa Nadi AE, Aiad AM, Mohamed SA, Soliman NAM. 2010. Replacement value of urea treated corn with cobs for concentrate feed mixture in pregnant ewes rations. J Anim Sci 6: 166-178.

13. Jackson MG 1978. Treating straw for animal feeding. Anim. Prod. Health. Paper $\mathrm{N}^{\circ}$ 10. Rome: FAO. [Internet]. Available in: http://www.fao.org/docrep/ 003/x6510e/X6510E00.htm\#TOC

14. Jeranyama P, Gracia A. 2004. Understanding relative feed value (RFV) and relative forage quality (RFQ). Cooperative Extension Service ExEx8149. South Dakota State Univer-sity. [Internet]. Available in: http:// pubstorage.sdstate.edu/ AgBio_Publications/articles/ ExEx8149.pdf

15. Lammers BP, Buckmaster DR, Heinrichs AJ. 1996. A simple method for the analysis of particle sizes of forage and total mixed rations. J Dairy Sci 79: 922-928. doi: 10.3168/jds.S00220302(96)76442-1

16. Oji UI, Mowat DN, Winch JB. 1977. Alkali treatment of corn stover to increase nutritive value. J Anim Sci 44:
798-802. doi: $10.2527 /$ jas $1977.445798 x$

17. Owen E. 1976. Farm wastes: straw, and another fibrous materials. In: Duckham AN, Jones JGW, Roberts EH (eds). Food production and consumption: the efficiency of human food chain and nutrient cycles. Amsterdam: North Holland Publ. p 299-318.

18. Ramírez GR, Aguilera JC, García G, Nuñez, AM. 2007. Effect of urea treatment on chemical composition and digestion of Cenchru sciliaris and Cynodon dactylon hays and Zea mays residues. J Anim Vet Adv 6: 1036-1041.

19. Sánchez E, Ortega C, Mendoza ME, Oziel G, Montanez D, Buntinx SE. 2012. Rastrojo de maíz tratado con urea y metionina protegida en dietas para ovinos en crecimiento. Interciencia 37(35): 395.

20. Shimada $A$. 2015. Nutricion animal. $3^{\text {a }}$ ed. México DF: Ed Trillas. 544 p.

21. Sundstol F, Coxwth E, Mowat DN. 1978. Improving the nutritive value of straw and other low quality roughages by treatment with ammonia. World Anim Rev 26: 13-21.

22. Tesfaye A, Chairatanayuth $P$, Vijchulata P. 2006. Effects of urea levels and treatment durations on chemical composition an in vitro dry matter digestibility of maize stover. Kasetsart J Nat Sci 40: 971-976.

23. Goering HK, Van Soest PJ. 1972. Forage fiber analysis. Agriculture Handbook N. ${ }^{\circ}$ 379. United States Department of Agriculture. 20 p. 